\title{
Effect of strontium ions on calcification of preosteoblasts cultured on porous calcium- and phosphate-containing titanium oxide layers formed by micro-arc oxidation
}

\author{
Mizuki SATO ${ }^{1}$, Peng CHEN², Yusuke TSUTSUMI², Makoto SHIOTA ${ }^{1}$, Takao HANAWA² and Shohei KASUGAI ${ }^{1}$ \\ ${ }^{1}$ Oral Implantology and Regenerative Dental Medicine, Tokyo Medical and Dental University, 1-5-45 Yushima, Bunkyo-ku, Tokyo 113-8510, Japan \\ ${ }^{2}$ Department of Metallic Biomaterials, Institute of Biomaterials and Bioengineering, Tokyo Medical and Dental University, 2-3-10 Kanda-Surugadai, \\ Chiyoda-ku, Tokyo 101-0062, Japan \\ Corresponding author, Peng CHEN; E-mail: chen.met@tmd.ac.jp
}

\begin{abstract}
Strontium (Sr) ions were added to calcium- and phosphate-containing porous titanium oxide layers formed by micro-arc oxidation (MAO) of titanium (Ti) substrates to improve their osseointegration. An MC3T3-E1 preosteoblast was used to evaluate the effect of the incorporated Sr species on cell calcification. Similar surface microporous morphologies of the oxide layers were observed for all specimens produced by MAO, while the contents of the incorporated Sr ions increased with increasing Sr concentrations in MAO electrolytes. The calcium- and phosphate-containing porous layers promoted the cell alkaline phosphatase (ALP) activity, while cell calcification was promoted by the Sr addition. In particular, the ALP activity significantly increased after 10 days of culture, and larger areas of calcified deposits were observed for the specimens treated with MAO electrolytes containing $0.15 \mathrm{~mol} \mathrm{~L}^{-1}$ of Sr species. The effect of Sr addition on the calcification of the MAO-treated Ti oxide layers was established in this study.
\end{abstract}

Keywords: Strontium, Micro-arc oxidation, Titanium, Osteogenic differentiation and calcification, MC3T3-E1

\section{INTRODUCTION}

Titanium (Ti) and its alloys have been used in dentistry as implant materials because of their good mechanical properties, high corrosion resistance, and low cytotoxicity. However, their poor bone fusion properties limit their clinical applications ${ }^{1}$. Various techniques have been explored to modify the surfaces of $\mathrm{Ti}$ and its alloys to improve their osseointegration, including plasma spraying, sol-gel method, and electrochemical deposition $^{2-12)}$. Among these modification methods, micro-arc oxidation (MAO) has attracted special attention since it is a relatively convenient and effective technique for depositing oxide coatings on the surfaces of nonferrous metals ${ }^{9-12)}$. More importantly, by using this technique, various desired elements can be introduced into the coated layers with porous structures ${ }^{11}$, such as calcium $(\mathrm{Ca})$, phosphorus $(\mathrm{P})$, magnesium $(\mathrm{Mg})$ and strontium (Sr), which are trace elements in the human body playing a significant role in bone formation.

$\mathrm{Sr}$ is considered a bioactive element that improves implant osseointegration. As was previously reported, $\mathrm{Sr}$ ions have been shown to enhance bone collagen synthesis and decrease bone resorption by inhibiting osteoclast resorbing activity ${ }^{13-20)}$. Therefore, it is expected that a local release of $\mathrm{Sr}$ ions from implant surfaces would directly improve implant osseointegration.

Since our previous studies have shown that MAO is a promising method to produce porous and firmly adherent bone formation concerned elements contained oxide deposition layers on metal substrates ${ }^{11)}$, it is

Color figures can be viewed in the online issue, which is available at J-STAGE.

Received Jan 22, 2016: Accepted Mar 24, 2016

doi:10.4012/dmj.2016-032 JOI JST.JSTAGE/dmj/2016-032 easy to optimize the $\mathrm{Sr}$ amounts introduced into the oxide deposition layers by altering MAO electrolyte compositions. Moreover, except for the first several days, a long-term stable release of metallic ions from the oxide deposition layers can be achieved by using the microporous structures formed during the MAO process $^{8)}$

In this study, Ti surfaces were modified by MAO using electrolytes with different $\mathrm{Sr}$ concentrations to promote calcification of preosteoblasts. Morphologies and elemental compositions of the surfaces as well as cross-sections of the $\mathrm{Ti}$ specimens obtained with and without MAO were characterized by scanning electron microscopy (SEM) and energy dispersive X-ray spectroscopy (EDS). A mouse preosteoblast MC3T3-E1 was utilized to study the calcification effect of Sr contents in the MAO-deposited layers on the Ti substrates in vitro. Osteogenic differentiation and calcified depositions of the MC3T3-E1 cells cultured on all specimens were evaluated using alkaline phosphatase (ALP) activity assay and alizarin red s staining, respectively.

\section{MATERIALS AND METHODS}

\section{Ti substrate preparation}

Commercially pure Ti discs (grade $2,8 \mathrm{~mm} \varphi \times 2.0 \mathrm{~mm}$ in thickness, Rare Metallic, Tokyo, Japan) were used in this study. Before the MAO treatment, Ti discs were mechanically polished with up to \#800-grid $\mathrm{SiC}$ abrasive paper and then ultrasonically rinsed in acetone and ethanol. The obtained specimens (not subjected to MAO) were named "BMAO" and stored in an auto-drying desiccator until further use. 
Table 1 Compositions of the electrolytes used for the MAO treatment of the Ti surfaces (M)

\begin{tabular}{cccc}
\hline Specimen name & Calcium acetate monohydrate & Strontium acetate hemihydrate & Calcium glycerophosphate \\
\hline Sr0M & 0.15 & 0 & 0.10 \\
Sr0.05M & 0.10 & 0.05 & 0.10 \\
Sr0.10M & 0.05 & 0.10 & 0.10 \\
Sr0.15M & 0 & 0.15 & 0.10 \\
\hline
\end{tabular}

\section{MAO treatment}

As reported in our previous work $^{10-12)}$, Ti substrates (BMAO) were fixed in a polytetrafluoroethylene holder, which was used as an anode, while a stainless steel cylindrical container served as the cathode. The electrolytes used for the MAO procedure were composed of calcium acetate monohydrate (Wako Pure Chemical Industries, Osaka, Japan), strontium acetate hemihydrate (Wako Pure Chemical Industries), and calcium glycerophosphate (Wako Pure Chemical Industries) (see Table 1). Both electrodes were connected to a DC power supply (PL-650-0.1, Matsusada Precision), while the treatment was performed at a positive voltage of $420 \mathrm{~V}$ and constant current of $12 \mathrm{~mA}$ for $10 \mathrm{~min}$. After the MAO treatment, all specimens were fully washed with distilled water and dried. All surface characterizations were performed for the MAO-treated areas.

\section{Surface characterization}

Images of the specimens produced with and without MAO were obtained using an Olympus SZX12 microscope (Olympus, Tokyo, Japan) at the same magnification, while their detailed surface morphology and compositions were investigated by scanning electron microscopy and energy dispersive X-ray spectroscopy (SEM/EDS, S-3400N, Hitachi High-Technologies, Tokyo, Japan).

\section{Cell cultures}

A mouse preosteoblast cell line (MC3T3-E1, RIKEN Bioresource Center, Ibaraki, Japan) was purchased and stored in a cell growth medium consisting of an alpha modification of the eagle's minimum essential medium ( $\alpha$-MEM, GIBCO, CA, USA) supplemented with $10 \%$ fetal bovine serum (FBS, GIBCO, NY, USA) and an antibiotic-antimycotic (100 $\mathrm{U} \mathrm{mL}^{-1}$ of penicillin, $100 \mathrm{mg}$ $\mathrm{mL}^{-1}$ of streptomycin, and $0.25 \mathrm{mg} \mathrm{mL}^{-1}$ of amphotericin B; GIBCO).

Before in vitro testing, all specimens were sterilized in 70 vol.\% ethanol for $20 \mathrm{~min}$ and thoroughly rinsed with deionized water. Then the cells were seeded onto the sterilized specimens with an approximate initial density of 5,000 cells $\mathrm{cm}^{-2}$. All the cell-containing specimens were incubated at $37^{\circ} \mathrm{C}$ in fully humidified atmosphere containing $5 \% \quad \mathrm{CO}_{2}$ in air. When the cells reached $100 \%$ confluence on the specimens, the osteogenic differentiation was induced, and this day was counted as 0 day. The growth medium was changed to a differentiation induction medium, where $2 \mathrm{mM}$ of $\beta$-glycerophosphate (Calbiochem, Darmstadt, Germany) and $50 \mathrm{mg} \mathrm{mL} \mathrm{m}^{-1}$ of L-ascorbic acid (Wako Pure Chemical Industries) were supplemented to the cell growth medium. This differentiation medium was changed every 3 days.

\section{Cell proliferation evaluation}

In order to evaluate the cell proliferation on the $\mathrm{Ti}$ specimens obtained with and without MAO, the number of attached cells was counted on each specimen using a cell counting kit (CCK-8, Dojindo Laboratories, Kumamoto, Japan) after 6, 10, and 14 days. The specimens were washed with a phosphate-buffered saline (PBS) buffer solution to remove any unattached cells, while the attached cells were harvested from each specimen and then assayed to obtain the cell number according to the instructions for the CCK- 8 kit. Sample absorbance was measured at a wavelength of $450 \mathrm{~nm}$ using a microplate reader (ChroMate Microplate Reader, Awareness Technology, Palm City, FL, USA).

\section{Osteogenic differentiation evaluation}

The ALP activity is very important for evaluating the osteogenic differentiation of cells ${ }^{21)}$. After 6, 10, and 14 days of culture, the MC3T3-E1 cells cultured on all specimens were washed twice with ice-cold PBS and then distributed by sonication (UD100, Tomy Seiko, Japan) on ice. The ALP activity of the cells was determined by LabAssay ${ }^{\text {TM }}$ ALP (Wako Pure Chemical Industries) according to the manufacturer's instructions, while their absorbance was measured at a wavelength of 405 nm using a ChroMate Microplate Reader (Awareness Technology).

\section{Calcification evaluation}

Calcification of the MC3T3-E1 cultures on the Ti specimens obtained with and without MAO was visually observed in the form of calcified deposits revealed by alizarin red s staining. After 14, 21, and 28 days of culture, the MC3T3-E1 cells cultured on all specimens were washed with ice-cold PBS and fixed with $4 \%$ formaldehyde. All specimens were stained with a $1 \%$ alizarin red $\mathrm{s}$ solution (adjusted to $\mathrm{pH}=4.2$ with ammonium hydroxide) at room temperature and then rinsed with ultra-pure water. After being fully dried, all specimens were photographed using a stereomicroscope (Olympus SZX12). In addition, the stained calcified areas were calculated as [(stained area/total dish area $) \times 100] \%$ 
using an image analyzing software (ImageJ, National Institutes of Health, Bethesda, MD, USA). The details of this procedure were described elsewhere ${ }^{22)}$.

\section{Statistical analysis}

All data were presented as mean \pm standard deviation of the mean. The obtained results were compared by one-way ANOVA followed by a Student-NewmanKeuls (SNK) test for multiple comparisons using SPSS Statistics 22 (SPSS, Chicago, IL, USA). Results with $p$ values $<0.05$ were deemed statistically significant.

\section{RESULTS}

\section{Specimen characterization}

The stereomicroscopic images of the specimens produced with and without MAO (Fig. 1) show that the microporous deposition coatings on the $\mathrm{Ti}$ specimens obtained by MAO are located in circular regions with diameters of $7 \mathrm{~mm}$ and characterized by a similar color regardless of the $\mathrm{Sr}$ concentrations in electrolytes. The areas outside the circular regions (Fig. 1b) may be a result of the leakage during treatment.

No significant difference in the surface microporous morphology were observed for the MAO-treated $\mathrm{Ti}$ specimens - even at different $\mathrm{Sr}$ concentrations in electrolytes (Figs. 2a-e). The results of the EDS analysis are presented in Fig. 2f. The obtained fractions of various elements indicate that with increasing the concentration of $\mathrm{Sr}$ in the electrolyte, its fraction on the surface of the microporous $\mathrm{Ti}$ oxide layer increases, while that of $\mathrm{Ca}$ decreases. Moreover, the observed changes in $\mathrm{Sr}$ electrolyte concentrations do not have a significant effect on the percentage concentrations of $\mathrm{P}$ on the surfaces of the microporous oxide layers.

The cross-sectional images of the specimens (Fig. 3) reveal that the MAO-deposited coating layers with an approximate thickness of $10 \mu \mathrm{m}$ are integrated with the Ti substrates. The EDS mapping images indicate that the $\mathrm{Ti}$ atoms originate from the substrate, the $\mathrm{O}$ atoms come from the oxide, and the $\mathrm{C}$ atoms originate from surface contamination; $\mathrm{Ti}, \mathrm{Ca}, \mathrm{P}$, and Sr elements were also detected in the oxide layers. Due to the rapid growth of microporous deposition during the MAO treatment, the electrolyte elements were incorporated into the oxide layers on the Ti substrates. More importantly, all elements (including $\mathrm{Sr}, \mathrm{Ti}, \mathrm{Ca}$, $\mathrm{P}$, and $\mathrm{O}$ ) were homogeneously distributed in these $\mathrm{Sr}$ containing microporous oxide layers, while increasing $\mathrm{Sr}$ electrolyte concentrations results in an increase in the EDS intensities of Sr.

\section{Cell proliferation}

The proliferation of the MC3T3-E1 cells cultured on all specimens was examined through the attached cell number on each specimen for the 6, 10, and 14-days cultures (Fig. 4). The number of the attached cells on all specimens increases with culture time. After 6 days of culture, the highest cell number was obtained for BMAO, whereas after 10 days culture, there was no

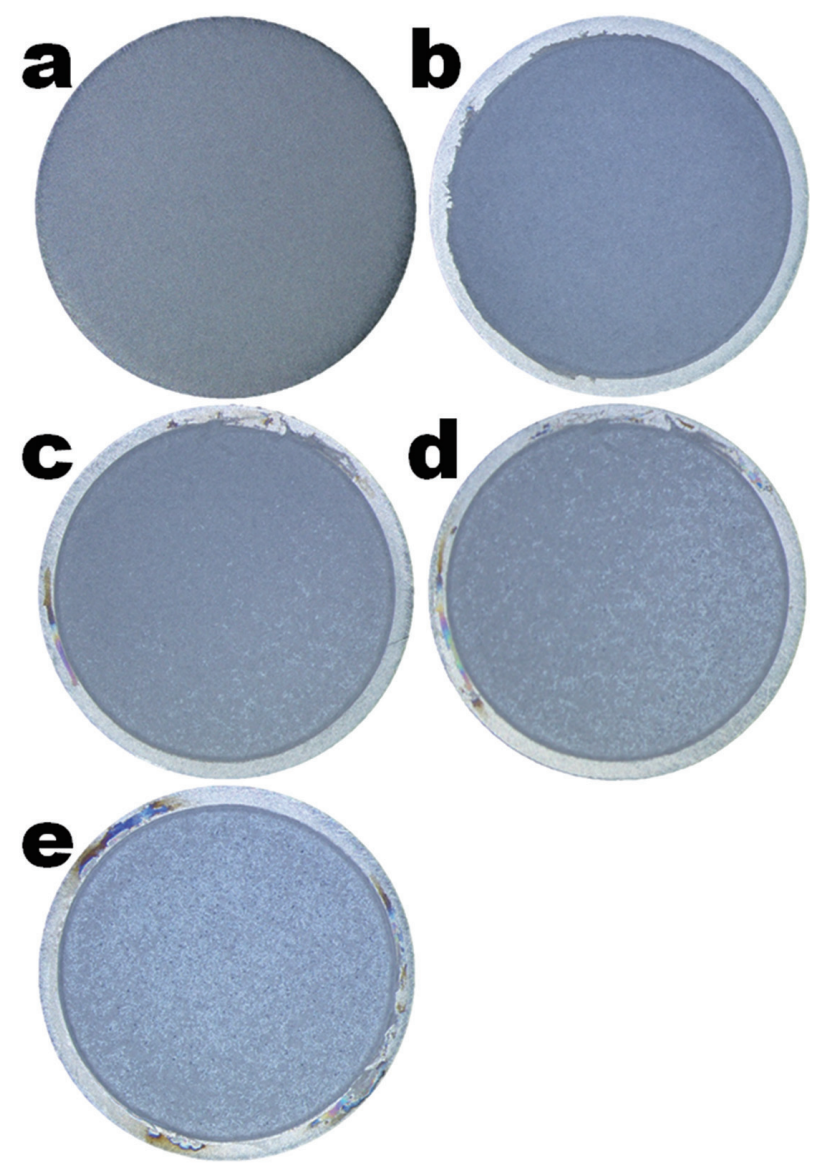

Fig. 1 Stereomicroscopic images of the specimens obtained with and without MAO.

Images of the specimens (a) before the MAO treatment (BMAO) and after the MAO treatment with the Sr concentrations of (b) $0 \mathrm{M}$ (SrOM), (c) $0.05 \mathrm{M}(\mathrm{Sr} 0.05 \mathrm{M})$, (d) $0.10 \mathrm{M}(\mathrm{Sr} 0.10 \mathrm{M})$, and (e) $0.15 \mathrm{M}(\mathrm{Sr} 0.15 \mathrm{M})$. The diameters of all specimens are $8 \mathrm{~mm} \varphi$, and the diameters of the MAO-treated areas are about $7 \mathrm{~mm} \varphi$.

significant difference in the attached cell numbers for all specimens. After 14 days of culture, a larger number of the attached cells were obtained for BMAO than for the MAO-treated specimens.

\section{Cell differentiation}

To evaluate the cellular osteogenic differentiation at different $\mathrm{Sr}$ concentrations in the oxide layers obtained by MAO, the intracellular ALP activity levels of the MC3T3-E1 cells cultured on all specimens were assayed after 6,10 , and 14 days of culture. The ALP activity levels increased for all specimens from the 6 to 14-days culture. After 6 days of culture, no significant differences were observed for the cells cultured on different specimens. However, higher levels of the ALP activity were detected for the cells cultured on the Sr0.15M specimen compared to the other specimens after 10 days 

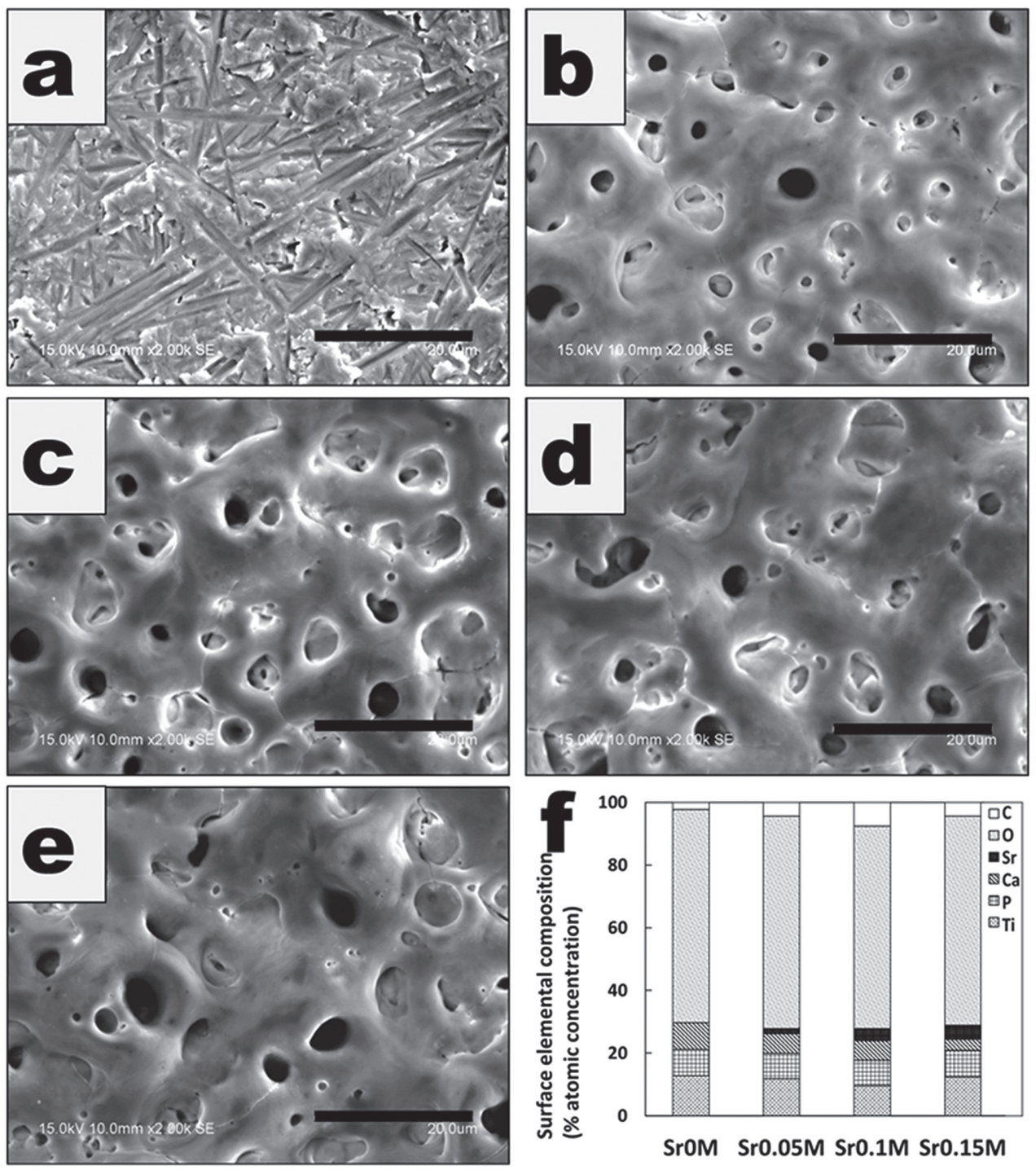

Fig. 2 SEM images and surface elemental compositions (\% atomic concentration) of all specimens obtained by SEM/EDS.

SEM images of (a) BMAO, (b) Sr0M, (c) Sr0.05M, (d) Sr0.10M, (e) Sr0.15M, and (f) surface elemental compositions determined by EDS.

of culture. After 14 days of culture, the cells cultured on the MAO-treated Ti specimens exhibited higher ALP activity levels compared to BMAO.

\section{Cell calcification}

Alizarin red s staining was used to present visible results of calcification for the MC3T3-E1 cells on all specimens after 21 and 28 days of culture. The calcified deposits observed for all specimens were stained with a crimson color (black color in the grayscale photos, Fig. 5a). However, compared with the calcified deposits on BMAO, the MAO-treated specimens were almost fully stained with the crimson color. The quantified results obtained by the ImageJ software show that a higher percentage of the calcified area was obtained for the MC3T3-E1 cells cultured on the Sr-containing microporous oxide coatings after 21 days of culture. Notably, the cells cultured on the Sr0.15M and Sr0.1M specimens produced the highest percentage of the calcified area among all the specimens after 28 days of culture.

\section{DISCUSSION}

In this study, different concentrations of Sr-containing microporous oxide layers were fabricated on the Ti specimens by MAO. Compared with other surface modification methods, the MAO treatment procedure employed in this study was a simple and easy way of producing $\mathrm{Ti}$ oxide surface coating layers containing 


\section{BMAO SrOM Sr0.05M Sr0.10M Sr0.15M}

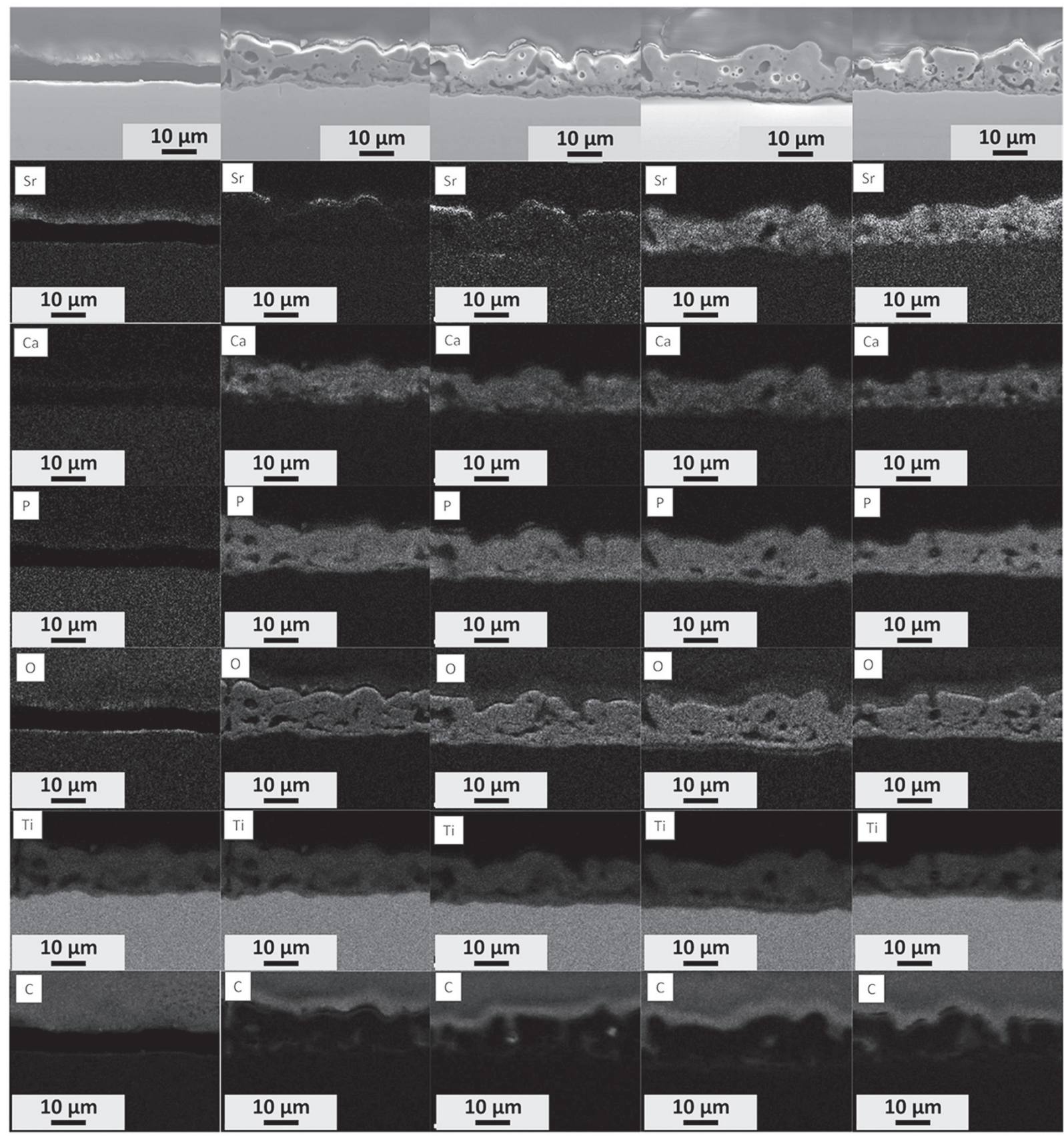

Fig. 3 Cross-sectional images of the specimens acquired by SEM and elemental maps of the specimens with multiple elements obtained by EDS.

specific target metal elements with the microporous structure. The microporous structures deposited on substrate surfaces by MAO can be controlled by varying operating voltage and current values ${ }^{11,13)}$; for example, a higher voltage leads to a larger pore diameter. In this study, the consistent conditions of the MAO treatment were used, except for the $\mathrm{Ca}$ and $\mathrm{Sr}$ concentrations. Therefore, similar surface microporous oxide layers were obtained for the Ti substrates even when the $\mathrm{Sr}$ concentration was varied (Figs. 2b-e). Because of the same MAO treatment time, similar thicknesses of the microporous oxide layers were observed for all specimens after the MAO treatment (Fig. 3).

Using the MAO technique, different $\mathrm{Sr}$ concentrations were obtained on/in the microporous oxide layers of the Ti specimens; more importantly, all 


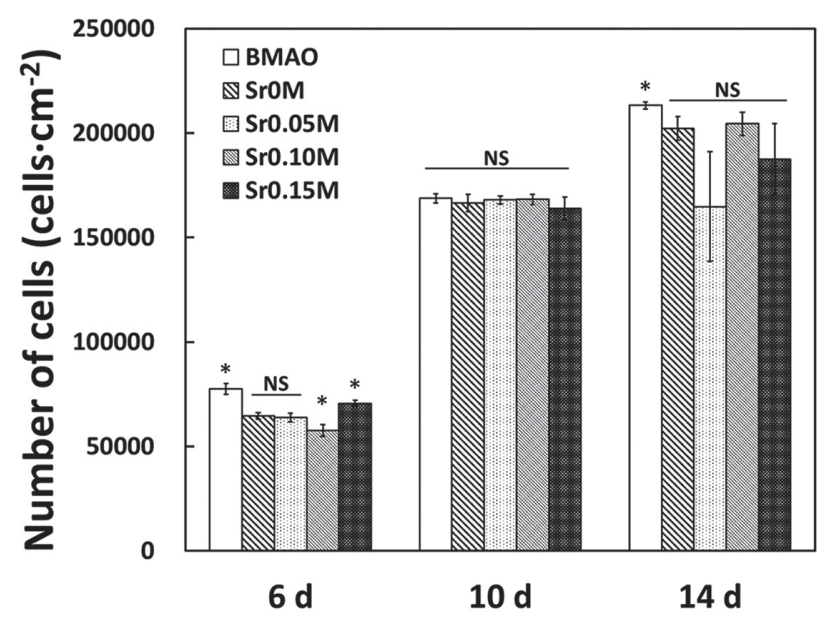

Fig. 4 Proliferation of the MC3T3-E1 cells on all specimens.

The attached cell numbers were counted using a cell counting kit-8. All values are presented as mean \pm standard deviation $(n \geq 3)$. The obtained data were compared by one-way ANOVA followed by a Student-Newman-Keuls test for multiple comparisons. ${ }^{*} p<0.05$; n.s. means non-significant.

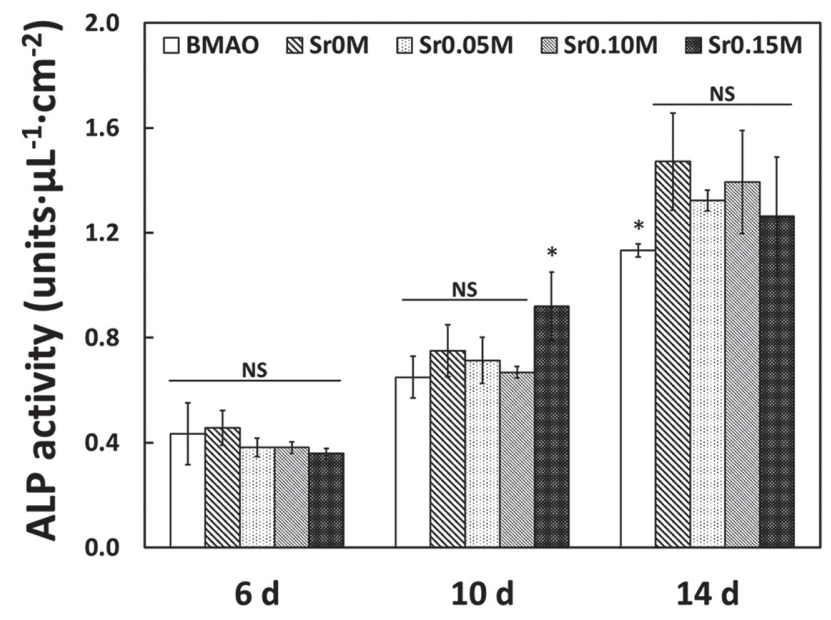

Fig. 6 ALP activity levels of the MC3T3-E1 cells on all specimens for the 6,10, and 14-days cultures.

All values are presented as mean \pm standard deviation ( $n \geq 3)$. The obtained data were compared by one-way ANOVA followed by a StudentNewman-Keuls test for multiple comparisons. ${ }^{*} p<0.05$; n.s. means non-significant.

the elements (including $\mathrm{Sr}, \mathrm{Ca}$, and $\mathrm{P}$ ) were uniformly distributed on/in these layers (Fig. 3). In addition, increasing $\mathrm{Sr}$ concentrations in electrolytes resulted in larger Sr contents on/in the microporous oxide layers (Figs. 2f and 3). Certainly, the surface microporous morphology and the thickness of the surface oxide layers can be easily controlled by changing the conditions of

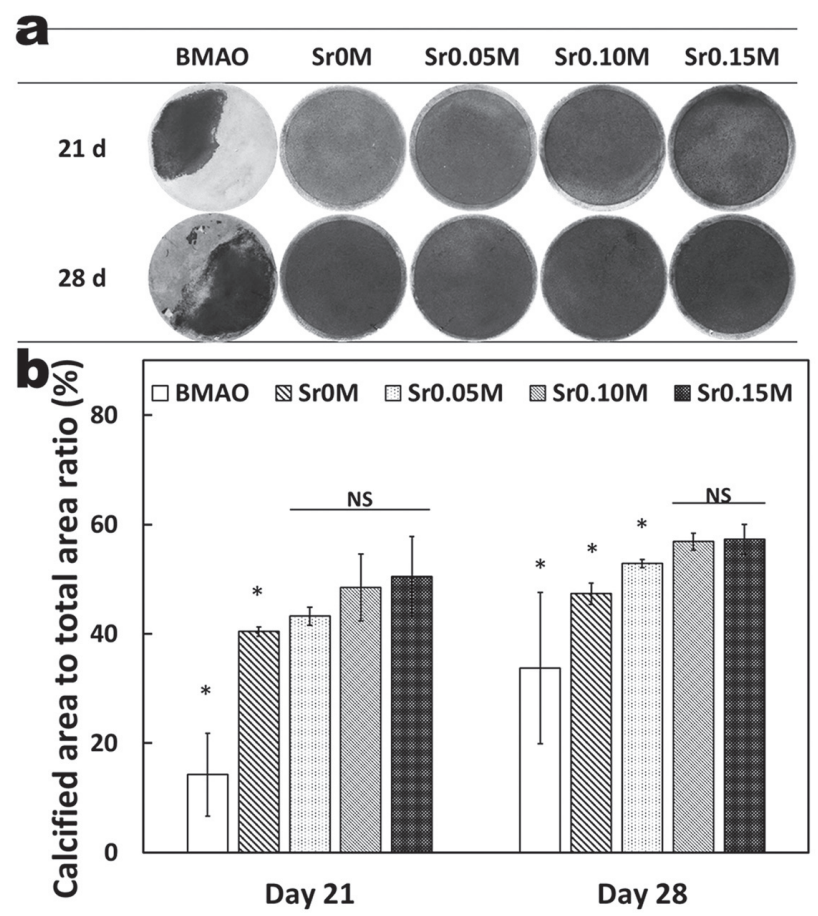

Fig. 5 Calcification evaluation of the MC3T3-E1 cells on all specimens for the 21 and 28-days cultures by alizarin red s staining.

(a) Grayscale photos of calcified deposits produced by the MC3T3-E1 cells on all specimens for the 21 and 28-days cultures after alizarin red s staining; (b) proportions of the calcified area to the total area for each specimen. All values are presented as mean \pm standard deviation $(n=3)$. The obtained data were compared by one-way ANOVA followed by a Student-Newman-Keuls test for multiple comparisons. ${ }^{*} p<0.05$; n.s. means non-significant.

the MAO treatment. However, the exact concentrations of $\mathrm{Sr}$ on/in these microporous oxide layers obtained by MAO are difficult to determine.

As the Ti specimens were surface-modified with the Sr-containing microporous oxide layers, an MC3T3-E1 preosteoblast was used to evaluate their potential effects on cell proliferation, differentiation, and calcification behaviors.

For the cell proliferation, no significant differences were observed for the specimens obtained by MAO (Fig. 4) indicating that adding different $\mathrm{Sr}$ concentrations to electrolytes (between 0 and $0.15 \mathrm{M}$ ) produces no significant effect on the cell proliferation. However, the MAO treatment procedure may itself have an effect on cell proliferation, as the number of the cells attached to the specimens obtained by MAO was less than that obtained for BMAO (Fig. 4). This phenomenon may be explained by a larger number of metal ions released from the microporous oxide layers, which may induce a higher cellular stress and influence the cellular signaling 
related to cell proliferation.

On the other hand, the incorporation of $\mathrm{Sr}, \mathrm{Ca}$, and $\mathrm{P}$ on/in the microporous oxide layers by the MAO treatment promotes cell osteogenic differentiation. Higher levels of the ALP activity were observed for the MC3T3-E1 cells on the microporous oxide layers after 14 days of culture (Fig. 6), which indicates larger osteogenic differentiation capacity. In particular, after 10 days of culture, a higher level of the ALP activity was observed for the cells cultured on the Sr0.15M specimen. Therefore, the incorporation of $\mathrm{Sr}$ should be beneficial for cell osteogenic differentiation - especially, for the MAO-treated specimens with electrolytes containing $0.15 \mathrm{M}$ of $\mathrm{Sr}$.

As the Sr-containing microporous oxide layers produced by MAO promote cell osteogenic differentiation, they may have an effect on the consequent cell calcification of preosteoblasts. The results obtained by the alizarin red s staining revealed that almost the whole MAO-treated areas were stained with a crimson color (Fig. 5a) indicating that the microporous oxide layers improved the hard tissue compatibility of the $\mathrm{Ti}$ substrates. This phenomenon was also confirmed by the ALP activity results obtained after 14 days of culture. More importantly, increasing $\mathrm{Sr}$ concentrations in electrolytes results in an increase in the incorporated $\mathrm{Sr}$ content on/in the microporous oxide layers and larger surface areas of the calcified deposits (Fig. 5b). Thus, increasing concentrations of the incorporated $\mathrm{Sr}$ on/in the microporous oxide layers improves osseointegration of the $\mathrm{Ti}$ substrates. In addition, similarly good osseointegration was observed for the Sr0.10M and Sr0.15M specimens.

The concentration of $\mathrm{Sr}$ incorporated on/in the microporous oxide layers on the Ti substrates is an important parameter for obtaining good osseointegration metal properties. It was reported that treatments with $0.1 \mathrm{mM}$ of $\mathrm{Sr}^{2+}$ promoted differentiation of osteoblast precursors into mature osteoblasts and increased the bone nodule number ${ }^{20)}$, which was also used in a clinical treatment; while a $0.12 \mathrm{mM}$ solution of $\mathrm{Sr}$ ranelate was used to treat blood active concentrations in postmenopausal osteoporotic patients ${ }^{18)}$. However, excessively high concentrations of $\mathrm{Sr}$ (up to $0.4 \mathrm{mM}$ ) delayed the effect on the osteoblastic differentiation with delayed expression on Cbfa1 and osteonectin, and the inhibitory effect on the bone sialoprotein expression $^{20)}$. In this study, MAO electrolytes with higher Sr concentrations were prepared. Compared with other surface modification methods, a better intake of target metal ions as well as stable and continuous ion release can be achieved through the MAO treatment. During the first several days of the cell attachment, higher Sr concentrations may be released from the surface of microporous deposition coatings; however, in the following several months, stable and lower $\mathrm{Sr}$ concentrations can be obtained from the inside of the coating layers through their microporous structure. Our data also exhibit improved osteogenic differentiation and calcification characteristics obtained for the MC3T3-E1 cells on the Sr0.15M specimen.

The results of this work show that adding $\mathrm{Ca}$ and $\mathrm{P}$ through $\mathrm{MAO}$ is beneficial for cell osteogenic differentiation, which is enhanced by incorporating $\mathrm{Sr}$ on/in microporous oxide layers. Moreover, these Srcontaining microporous oxide layers also promote cell calcification, which is important for boosting metal implant osseointegration.

\section{CONCLUSION}

In this study, $\mathrm{Sr}$ was added to the microporous $\mathrm{Ti}$ oxide layers containing $\mathrm{Ca}$ and phosphates species formed by MAO on the Ti substrates to improve their osseointegration. Increasing $\mathrm{Sr}$ concentrations in electrolytes produces no effect on the microporous structure of the oxide layers deposited on the $\mathrm{Ti}$ specimens, while the concentration of the incorporated $\mathrm{Sr}$ ions was increased. In addition, the incorporated $\mathrm{Ca}$, phosphates, and $\mathrm{Sr}$ species were homogeneously distributed on/in these microporous oxide layers. Although the MAO-treated oxide layers on the $\mathrm{Ti}$ substrates produce a small inhibitory effect on cell proliferation (compared with that observed for the BMAO specimen), they also promote cell osteogenic differentiation. The calcified deposits stained by alizarin red s show that the Sr-containing microporous oxide layers promote calcification of MC3T3-E1 cells (especially on the MAO-treated $\mathrm{Ti}$ specimens) using electrolytes with Sr concentrations of 0.1 and $0.15 \mathrm{M}$. The obtained results suggest that the specimens treated with MAO electrolytes containing $0.15 \mathrm{~mol} \mathrm{~L}^{-1}$ of $\mathrm{Sr}$ can be beneficial for cell osteogenic differentiation and calcification. The effect of $\mathrm{Sr}$ addition on the calcification of the MAO-treated Ti substrates was clearly revealed. Our study provides an effective surface modification strategy for achieving better implant osseointegration of metallic biomaterials.

\section{ACKNOWLEDGMENTS}

This work was partially supported by a Grant-in-Aid for Scientific Research (Kiban A: No. 22240059) from the Ministry of Education, Culture, Sports, Science, and Technology of Japan.

\section{REFERENCES}

1) Tengvall P. Proteins at titanium interfaces. In: Brunette DM, Tengvall P, Textor M, Thomsen P, editor. Titanium in medicine: Material science, surface science, engineering, biological responses \& medical applications. 1st ed. Berlin: Springer; 2001. p. 457-672.

2) Yang YC, Chan E, Hwang BH, Lee SY. Biaxial residual stress states of plasma-sprayed hydroxyapatite coatings on titanium alloy substrate. Biomaterials 2000; 21: 1327-1337.

3) Zheng X, Huang M, Ding C. Bond strength of plasma-sprayed hydroxyapatite/Ti composite coatings. Biomaterials 2000; 21 : 841-849.

4) Hsieh MF, Perng LH, Chin TS. Hydroxyapatite coating on Ti6Al4V alloy using a sol-gel derived precursor. Mater Chem Phys 2002; 74: 245-250. 
5) Milella E, Cosentino F, Licciulli A, Massaro C. Preparation and characterization of titania/hydroxyapatite composite coatings obtained by sol-gel process. Biomaterials 2001; 22: 1425-1431.

6) Abe Y, Hiasa K, Takeuchi M, Yoshida Y, Suzuki K, Akagawa Y. New surface modification of titanium implant with phospho-amino acid. Dent Mater J 2005; 24: 536-540.

7) Zhang QY, Leng Y, Xin R. A comparative study of electrochemical deposition and biomimetic deposition of calcium phosphate on porous titanium. Biomaterials 2005; 26: 2857-2865.

8) Huang Q, Liu X, Elkhooly TA, Zhang R, Yang X, Shen Z, Feng Q. Preparation and characterization of TiO2/silicate hierarchical coating on titanium surface for biomedical applications. Mater Sci Eng C Mater Biol Appl 2016; 60: 308316.

9) Song WH, Jun YK, Han Y, Hong SH. Biomimetic apatite coatings on micro-arc oxidized titania. Biomaterials 2004; 25 : 3341-3349.

10) Nyan M, Tsutsumi Y, Oya K, Doi H, Nomura N, Kasugai S, Hanawa T. Synthesis of novel oxide layers on titanium by combination of sputter deposition and micro-arc oxidation techniques. Dent Mater J 2011; 30: 754-761.

11) Ma C, Nagai A, Yamazaki Y, Toyama T, Tsutsumi Y, Hanawa T, Wang W, Yamashita K. Electrically polarized micro-arc oxidized TiO2 coatings with enhanced surface hydrophilicity. Acta Biomater 2012; 8: 860-865.

12) Tsutsumi Y, Niinomi M, Nakai M, Tsutsumi H, Doi H, Nomura N, Hanawa T. Micro-arc oxidation treatment to improve the hard-tissue compatibility of Ti-29Nb-13Ta-4.6Zr alloy. Appl Surf Sci 2012; 262: 34-38.

13) Ma WH, Liu YJ, Wang W, Zhang YZ. Improved biological performance of magnesium by micro-arc oxidation. Braz J Med Biol Res 2015; 48: 214-225.

14) Capuccini C, Torricelli P, Sima F, Boanini E, Ristoscu C, Bracci B, Socol G, Fini M, Mihailescu IN, Bigi A. Strontiumsubstituted hydroxyapatite coatings synthesized by pulsed- laser deposition: In vitro osteoblast and osteoclast response. Acta Biomater 2008; 4: 1885-1893.

15) Sakai A, Valanezahad A, Ozaki M, Ishikawa K, Matsuya S. Preparation of Sr-containing carbonate apatite as a bone substitute and its properties. Dent Mater J 2012; 31: 197205.

16) Ni GX, Chiu KY, Lu WW, Wang Y, Zhang YG, Hao LB, Li ZY, Lam WM, Lu SB, Luk KD. Strontium-containing hydroxyapatite bioactive bone cement in revision hip arthroplasty. Biomaterials 2006; 27: 4348-4355.

17) Sila-Asna M, Bunyaratvej A, Maeda S, Kitaguchi H, Bunyaratavej N. Osteoblast differentiation and bone formation gene expression in strontium-inducing bone marrow mesenchymal stem cell. Kobe J Med Sci 2007; 53: 25-35.

18) Meunier PJ, Roux C, Seeman E, Ortolani S, Badurski JE, Spector TD, Cannata J, Balogh A, Lemmel EM, PorsNielsen S, Rizzoli R, Genant HK, Reginster JY. The effects of strontium ranelate on the risk of vertebral fracture in women with postmenopausal osteoporosis. New Engl J Med 2004; 350: 459-468.

19) Qiu K, Zhao XJ, Wan CX, Zhao CS, Chen YW. Effect of strontium ions on the growth of ROS17/2.8 cells on porous calcium polyphosphate scaffolds. Biomaterials 2006; 27: 1277-1286.

20) Bonnelye E, Chabadel A, Saltel F, Jurdic P. Dual effect of strontium ranelate: Stimulation of osteoblast differentiation and inhibition of osteoclast formation and resorption in vitro. Bone 2008; 42: 129-138.

21) Umezawa T, Chen P, Tsutsumi Y, Doi H, Ashida M, Suzuki S, Moriyama K, Hanawa T. Calcification of MC3T3-E1 cells on titanium and zirconium. Dent Mater J 2015; 34: 713-718.

22) Chen P, Nagai A, Tsutsumi Y, Ashida M, Doi H, Hanawa T. Differences in the calcification of preosteoblast cultured on sputter-deposited titanium, zirconium, and gold. J Biomed Mater Res A 2016; 104: 639-651. 